\title{
Outcomes of Anti-Tubercular Treatment Among Patients with Ocular Tuberculosis Seen in A Tertiary Government Hospital in The Philippines
}

\author{
Marc Alfred C Mangahas*, Cris Martin P Jacoba, Isa M Jacoba, Jessica Marie R Abano, Juan S Lopez and Pia \\ Regina E Galvante
}

Department of Health Eye Center, East Avenue Medical Center, East Avenue Medical Center, Diliman, Quezon City

*Corresponding author: Marc Alfred C Mangahas, Department of Health Eye Center, East Avenue Medical Center, East Avenue Medical Center, Diliman, Quezon City.
Received Date: August 11, 2020

Published Date: October 23, 2020

\begin{abstract}
Objective: To evaluate the treatment outcomes of ocular tuberculosis (OTB) patients who underwent anti-tubercular treatment.

Methods: A retrospective chart review of OTB patients seen in a tertiary government hospital in the Philippines in over 10 years who underwent at least 6 months of anti-tubercular treatment (ATT) and 6 months follow up after treatment. The main outcome was the success or failure of ATT in terms of ability to taper down oral steroids to less than $10 \mathrm{mg} /$ day or topical steroids to twice a day. Factors such as patient demographics, ocular presentation, classification, diagnostic score, and duration of treatment associated with the outcomes were correlated.

Results: Nineteen patients who received atleast 6 months ATT and 6 months follow-up were involved in the study. The mean age was 41+/12.476. Most subjects were male ( $n=15,71.4 \%)$. Most patients were classified as possible OTB $(n=16,88.9 \%)$ and about half presented as panuveitis $(n=11,52.4 \%)$. Treatment success was seen in majority of the patients $(n=15,78.9 \%)$. Most common complication noted was cataract formation.
\end{abstract}

Conclusion: Anti-tubercular treatment of at least 6 months showed good clinical outcomes in OTB patients with minimal complications.

\section{Introduction}

Tuberculosis (TB) continues to be one of the leading morbidities in the last decade worldwide [1]. The Philippines remains to be one of five countries contributing to $56 \%$ of the total number of people affected [1]. In 2016, there were an estimated 1 million prevalent cases and 570,000 incident cases [1]. A report on uveitis patients seen in a tertiary government hospital showed ocular tuberculosis (OTB)as one of the most common diagnosis [2]. However, up to $60 \%$ of patients who have ocular OTB may not be diagnosed to have pulmonary TB [3]. TB infection affects any part of the eye leading to mild temporary blurring of vision to severe permanent visual loss. It can present with non-specific ocular symptoms and signs earning it the title of the "great mimicker". It poses as a treatment conundrum to ophthalmologists due to the lack of standardized diagnostic and treatment guidelines [4-5]. The current standard for diagnosing OTB, microbiologic evidence from ocular samples, is often expensive and have may poor yield [4] thus, ophthalmologists are left to presume the diagnosis based on corroborative ocular signs (i.e broad based posterior synechiae, retinal perivasculitis with or without discrete choroiditis/scars, multifocal serpiginoid choroiditis, choroidal granuloma) and systemic tests (i.e. tuberculin skin test (TST), tuberculosis blood test or Interferon Gamma Release Assay (IGRA) and chest radiographs). Even so, the management is only arbitrary [4-5]. Steroids and anti-tubercular therapy have been reported to be beneficial [4-14]. Reports show 
that steroids help control inflammation and adjuvant anti-tubercular therapy reduces recurrence [4-14]. However, a definite protocol for time of initiation and duration is lacking.

Locally, there is no study evaluating the response and effectiveness of anti-tubercular treatment on patients with presumed ocular tuberculosis. Standard treatment guidelines cannot be fully established due to lack of data. This study is aimed to report on the demographics, clinical presentation, time of initiation and duration of treatment of OTB patients managed in a tertiary government hospital using anti-tubercular regimen with adjuvant steroids.

\section{Methodology}

This was a retrospective chart review of all OTB patients seen at a uveitis clinic in a tertiary government hospital from 2004 to August 2018. It adhered to the basic principles of the Declaration of Helsinki and conformed to the guidelines set forth by the International Council for Harmonisation - Good Clinical Practice (ICHGCP). As such, it was approved by the institutional ethics review board prior to implementation of the research. The authors declare no patient personal data or information leading to their identification appears in this study.

Patients included in the study fulfilled the following: 1)At least one clinical sign suggestive of OTB 2) Microbiologic confirmation of Mycobacterium tuberculosis from ocular fluids or tissues OR Evidence of chest radio graphs consistent with TB infection or clinical evidence of extraocular TB OR Microbiological confirmation from sputum or extraocular sites (documented exposure to TB, immunological evidence $\mathrm{Tb}$ infection i.e. TST $>10 \mathrm{~mm},(+)$ tuberculosis blood test or Interferon Gamma Release Assay (IGRA) [4-5] 3)at least 6 months of anti-tubercular treatment and at least 6 months follow up with Uveitis clinic. Patients who were diagnosed with other ocular disorders that may cause uveitis or those who underwent prior ocular surgery 3 months or less prior to diagnosis of OTB were excluded from the study.

Information on patient demographics, clinical findings, diagnostics, and therapeutic protocol were encoded into a data information sheet. Initial visual acuity, anterior and posterior chamber inflammation and retinal lesion progression/regression were reviewed. Patients were then classified using Gupta's diagnostic classification of OTB [8]. They were also graded using Lopez's proposed diagnostic scoring criterion. (3 points for Healed TB scar on radiograph, 2 points for TST,+ 1 point Living in household with active $\mathrm{TB}+, 2$ points characteristic clinical lesion) [6]. Details of frequency, dosage of topical and oral steroid were also encoded. The duration of ATT was reviewed. The primary outcome measure was treatment success or failure. Treatment success was considered when oral steroids was tapered to less than $10 \mathrm{mg} /$ day or topical steroids to less than twice a day within 6 months after anti-tubercular treatment. Treatment failure was considered when these requirements were not achieved. Factors associated with treatment success or failure (i.e. age, sex, clinical presentation at time of onset, timing and duration of anti-tubercular treatment, diagnostic classification and score) were also noted. Complications occurring during the course of ATT and 6 months after ATT were reviewed. Qualitative variables were presented and analyzed as percentages. Parameters such as demographics, OTB classification, ocular involvement and OTB diagnostic scoring were correlated to treatment failure or success using either Fisher's exact test or a Chi-square test.

\section{Results}

In a span of 10 years 81 patients were initially diagnosed as cases of ocular tuberculosis. Of the 81 patients seen, 7 patients were discovered to be of a different uveitic cause. Of the remaining 73 patients, 50 failed to qualify for the study due to insufficient follow up or failure to initiate treatment. Five more patients were excluded due to already ongoing ATT regimen. Charts of eighteen patients were reviewed. The mean age was 40.8+/-16.7. Most subjects were male $(n=13,72.2 \%)$. Majority of patients were diagnosed as possible OTB with an average diagnostic score of 4.8 based on Lopez criteria [7]. About half presented as pan uveitis ( $\mathrm{n}=11,52.4 \%)$. Bilateral involvement was noted in almost half of the subjects $(n=10$, $47.6 \%)$.

Majority of the patients underwent only 6 months of ATT. Consequently, most successful cases were at 6 months $(n=15,71.5 \%)$. Time of treatment initiation usually started 1-2 months from diagnosis and showed favorable outcomes. Five cases were classified as failure ( $n=4,6$ months ATT, $n=1,9$ months ATT). Clinical presentation ( $p<0.538)$, diagnostic classification $(p<0.524)$, and treatment duration $(p<0.490)$ showed the closest correlation to treatment outcome. The most common complication encountered was cataract $(n=8,44.4 \%)$.

\section{Discussion}

Our study showed that in the a 10-year span, more than half of patients with OTB did not receive ATT. Most of these patients only had one consult or were lost to follow-up before treatment could be initiated. Another portion of patients did not present with radiologic evidence of pulmonary tuberculosis or clinically active pulmonary TB. This causes hesitation in the initiation of ATT by other clinicians. Patients who receive ATT often had more objective evidence of TB infection such as active clinical signs of pulmonary TB, chest radiograph findings, a positive sputum smear or were already diagnosed with pulmonary tuberculosis. We recommended that ophthalmologists should work in coordination with pulmonary or infectious disease service in regards to managing OTB patients.

Diagnosis and management of OTB remains a conundrum due non-specific signs and a lack of diagnostic criteria. In our study, patients were diagnosed as ОТВ by correlating history, ocular signs and symptoms and diagnostic findings. On review, all patients fulfilled the diagnostic classification proposed by Gupta8 and scored $>4$ points in the diagnostic scoring by Lopez [7]. As previously 
stated, a patient's clinical presentation, Gupta's classification and duration of treatment showed a likely correlation to control and non-recurrence ocular inflammation. The "Gupta classification" and "Lopez diagnostic scoring" may be considered helpful tools to aid in the systematic approach to diagnosing and managing OTB. Further validation however of these clinical tools is still recommended. The non-recurrence of inflammation after ATT found in our study ( $72 \%$

Table 1: Gupta Classification of intraocular tuberculosis ${ }^{8}$

\begin{tabular}{|c|c|}
\hline Clinical diagnostic group & Case definition criteria \\
\hline \multirow{2}{*}{$\begin{array}{c}\text { Confirmed IOTB (both } 1 \\
\text { and 2) }\end{array}$} & 1. At least one clinical sign suggestive of IOTB \\
\hline & 2. Microbiological confirmation of Mycobacterium tuberculosis (MTB) from ocular fluids/tissues \\
\hline \multirow{5}{*}{$\begin{array}{l}\text { Probable IOTB }(1,2 \text {, and } \\
\quad 3 \text { together })\end{array}$} & 1. At least one clinical sign suggestive of IOTB (and other etiologies excluded) \\
\hline & $\begin{array}{l}\text { 2. Evidence of chest X-ray consistent with TB infection or clinical evidence of extraocular TB or microbiological confirma- } \\
\text { tion from sputum or extraocular sites }\end{array}$ \\
\hline & 3. At least one of the following: \\
\hline & a) Documented exposure to TB \\
\hline & b) Immunological evidence TB infection \\
\hline \multirow{6}{*}{$\begin{array}{l}\text { Possible IOTB (1, 2, and } \\
3 \text { together) (or } 1 \text { and } 4)\end{array}$} & 1. At least one clinical sign suggestive of IOTB (and other etiologies excluded) \\
\hline & 2. Chest X-ray not consistent with TB infection and no clinical evidence of extraocular TB \\
\hline & 3. At least one of the following: \\
\hline & a. Documented exposure to TB \\
\hline & b. Immunological evidence TB infection \\
\hline & $\begin{array}{l}\text { 4. Evidence of chest X-ray consistent with TB infection or clinical evidence of extraocular TB but none of the characteris- } \\
\text { tics given in } 3\end{array}$ \\
\hline
\end{tabular}

Table 2: Lopez Diagnostic Scoring

\begin{tabular}{|c|c|c|c|c|c|c|}
\hline \multicolumn{4}{|c|}{ Criteria } & \multicolumn{3}{|c|}{ Score } \\
\hline \multicolumn{4}{|c|}{ Healed TB scar on radiograph } & \multicolumn{3}{|c|}{3} \\
\hline \multicolumn{4}{|c|}{ Tuberculin Skin Test } & \multicolumn{3}{|c|}{2} \\
\hline \multicolumn{4}{|c|}{ Living in household with active TB } & \multicolumn{3}{|c|}{1} \\
\hline \multicolumn{4}{|c|}{ Characteristic clinical lesion } & \multicolumn{3}{|c|}{2} \\
\hline Computed Cut-off score & Sensitivity & specificity & $\mathbf{L R}+$ & LR- & AUC & P-Value \\
\hline$>3$ & 92.49 & 66.67 & 2.78 & 0.31 & $0.88(95 \% \mathrm{CI}, 0.772$ to 0.951$)$ & 0.0001 \\
\hline \multicolumn{7}{|c|}{ LR+ positive likelihood ratio, LR- negative likelihood ratio, AUC area under the curve, CI confidence interval } \\
\hline & & $\begin{array}{r}\mathrm{S} \\
\text { ns N }\end{array}$ & $\begin{array}{l}\text { ificant } \\
\text { ignific }\end{array}$ & & & \\
\hline
\end{tabular}

Table 3: Patient demographics

Table 3: Patient demographics
\begin{tabular}{|c|c|c|}
\hline \multicolumn{2}{|c|}{ No. of patients } & $\mathbf{1 8}$ \\
\hline \multirow{2}{*}{ Age } & Mean (SD) & $40.8(16.7)$ \\
\hline \multirow{3}{*}{ Sex } & Range & $19-84$ \\
\cline { 2 - 3 } & Male & $13(72.2 \%)$ \\
\cline { 2 - 3 } & Female & $5(27.8 \%)$ \\
\hline Clinical presentation & Anterior & $1(5.6 \%)$ \\
\hline \multirow{3}{*}{ Laterality } & Intermediate & $1(5.6 \%)$ \\
\cline { 2 - 3 } & Posterior & $6(33.3 \%)$ \\
\cline { 2 - 3 } & Pan uveitis & $10(55.6 \%)$ \\
\cline { 2 - 3 } & Right & $7(38.9 \%)$ \\
\cline { 2 - 3 } & Left & $2(11.1 \%)$ \\
\hline
\end{tabular}

non-recurrence) is consistent with findings of Figueira in their meta-analysis (84\% non-recurrence) [6]. This response would further validate the diagnosis of OTB in patients who underwent ATT. Our study showed that at least 6 months of anti-tubercular treatment was sufficient in controlling of OTB. This in contrast to other studies recommending that treatment should be extended to 9 months or more [8-15]. 
Table 4: Correlation of Factors with Treatment Outcome

\begin{tabular}{|c|c|c|c|c|}
\hline \multirow{2}{*}{\multicolumn{2}{|c|}{$\begin{array}{c}\text { Factor } \\
\text { No of patients }\end{array}$}} & \multicolumn{2}{|c|}{ Outcome } & \multirow[t]{2}{*}{ p-value } \\
\hline & & Success & Failure & \\
\hline \multicolumn{2}{|r|}{$\begin{array}{c}\text { No of patients } \\
13 \\
\end{array}$} & 5 & & \\
\hline Age & Mean (SD) & $40.8(18.6)$ & $41.0(12.5)$ & 0.98 \\
\hline \multirow{3}{*}{ Sex } & Range & $19-22$ & $22-52$ & \\
\hline & Male & $9(69.2 \%)$ & $4(30.8 \%)$ & 1 \\
\hline & Female & $4(80.0 \%)$ & $1(20.0 \%)$ & \\
\hline \multirow{4}{*}{ Clinical presentation } & Anterior & $1(100 \%)$ & - & 0 \\
\hline & Intermediate & - & $1(100 \%)$ & 0.538 \\
\hline & Posterior & $5(83.3 \%)$ & $1(16.7 \%)$ & \\
\hline & Panuveitis & $7(70.0 \%)$ & $3(30.0 \%)$ & \\
\hline \multirow{4}{*}{$\begin{array}{l}\text { Time of initiation of } \\
\text { TB treatment }\end{array}$} & $<1$ month & $4(66.7 \%)$ & $2(33.3 \%)$ & 1 \\
\hline & $1-2$ months & $5(71.4 \%)$ & $2(28.6 \%)$ & \\
\hline & 3-6 months & $3(75.0 \%)$ & $1(25.0 \%)$ & \\
\hline & $>6$ months & $1(100 \%)$ & - & \\
\hline \multirow{3}{*}{$\begin{array}{c}\text { Duration of TB treat- } \\
\text { ment }\end{array}$} & 6-7 months & $6(60.0 \%)$ & $4(40.0 \%)$ & 0.524 \\
\hline & 8-9 months & $3(75.0 \%)$ & $1(25.0 \%)$ & \\
\hline & $>10$ months & $4(100.0 \%)$ & - & \\
\hline \multirow{2}{*}{$\begin{array}{c}\text { Diagnostic classifica- } \\
\text { tion }\end{array}$} & Probable & $1(50.0 \%)$ & $1(50.0 \%)$ & 0.49 \\
\hline & Possible & $12(75.0 \%)$ & $4(25.0 \%)$ & \\
\hline \multirow{2}{*}{ Score } & Mean (SD) & $4.8(1.1)$ & $4.8(1.3)$ & 0.96 \\
\hline & Range & Apr-62 & 24-Apr & \\
\hline
\end{tabular}

Table 5: Complications

\begin{tabular}{|c|c|c|}
\hline Complication type & No of patients & \% \\
\hline Cataract & 8 & 44.4 \\
\hline Ocular Hypertension & 1 & 5.6 \\
\hline Neovascular Glaucoma & 1 & 5.6 \\
\hline
\end{tabular}

To our knowledge, our study is the first of its kind to show demographics as well as outcomes of anti-tubercular therapy on OTB patients in the Philippines. The data gathered may be the basis for future studies and formation of diagnostic and treatment guidelines of OTB. Statistically, no significant correlation was seen between treatment outcome and patient sex, age, involvement, duration and time of initiation. A larger population is recommended to validate a correlation to treatment outcomes. In conclusion, anti-tubercular treatment of at least 6 months with adjuvant topical or oral steroids showed good outcomes in terms of decreasing inflammation and reduction of recurrence. Prompt diagnosis and patient education is recommended to ensure timely initiation of treatment and follow-up.

\section{Acknowledgement}

None.

\section{Conflict of Interest}

No Conflict of Interest.

\section{References}

1. (2017) Global tuberculosis report 2017. Geneva: World Health Organization

2. Abaño J, Galvante P, Siopongco P, Dans K, Lopez J (2017) Review of Epidemiology of Uveitis in Asia: Pattern of Uveitis in a Tertiary Hospital in the Philippines. Ocul Immunol Inflamm 25(sup1): S75-S80.

3. V Paul Chan Ocular Tuberculosis (TB) - Middle East/North Africa Retrieved from https://www.aao.org/topic-detail/ocular-tuberculosistb-asia-pacific

4. Agrawal R, Gupta B, Gonzalez Lopez JJ, Farzana Rahman, Sumita Phatak, et al. (2015) The role ofanti-tubercular therapy in patients with presumed ocular tuber-culosis. Ocul Immunol Inflamm 23(1): 40-46.

5. Gupta V, Gupta A, Rao NA (2007) Intraocular tuberculosis an update. Surv Ophthalmol 52(6): 561-587. 
6. Figueira L, Fonseca S, Ladeira I, Duarte R (2017) Ocular tuberculosis Position paper on diagnosis and treatment management. Rev Port Pneumol 23(1): 31-38.

7. J Lopez (2017) Validation of A Proposed Diagnostic Criteria for Intraocular Tuberculosis A Retrospective Evaluation. (Presented at Luasanne International Ocular Inflammation Society, Switzerland, October 20, 2017)

8. Ang M, Hedayatfar A, Wong W, Chee SP (2012). Duration of antitubercular therapy in uveitis associated with latent tuberculosis: a casecontrol study. Br J Ophthalmol 96(3): 332-336.

9. Gupta A, Sharma A, Bansal R, Sharma K (2015) Classification of intraocular tuberculosis. Ocul Immunol Inflamm 23(1): 7-13.

10. Basu S, Nayak S, Padhi TR, Das T (2013) Progressive ocular inflammation following anti-tubercular therapy for presumed ocular tuberculosis in a high-endemic setting. Eye (Lond) 27(5): 657-662.

11. Lee C, Agrawal R, Pavesio C (2016) Ocular tuberculosis a clinical conundrum. Ocul Immunol Inflamm 24(2): 237-242.
12. Manousaridis K, Ong E, Stenton C, Gupta R, Browning AC, et al. (2013) Clinical presentation, treatment, and outcomes in presumedintraocular tuberculosis: experience from Newcastle uponTyne, UK. Eye (London) 27(4): 480-486.

13. Lou SM, Montgomery PA, Larkin KL, Winthrop K, Zierhut M, et al. (2015) Diagnosis and treatment for ocular tuberculosis among uveitis specialists: the international perspective. Ocul Immunol Inflamm 23(1): 32-39.

14. Bansal, Reema et al. nRole of Anti-Tubercular Therapy in Uveitis With Latent/Manifest Tuberculosis. American Journal of Ophthalmology 146(5): 772-779.

15. Lara LPR, Ocampo V (2013) Prevalence of presumed ocular tuberculosis among pulmonary tuberculosis patients in a tertiary hospital in the Philippines. J Ophthalmic Inflamm Infect 3: 1 